\title{
CARACTERIZAÇÃo E AVALIAÇÃo CATALÍTICA DE ÓXIDOS MISTOS V-Mg-Al NA REAÇÃO DE DECOMPOSIÇÃO DO ISOPROPANOL.
}

\author{
F. A. de SIPOLLI ${ }^{1}$ e G. G. CORTEZ, ${ }^{1, *}$ \\ ${ }^{1}$ Escola de Engenharia de Lorena - USP, Departamento de Engenharia Química \\ E-mail para contato: cortez@dequi.eel.usp.br
}

\begin{abstract}
RESUMO - Precursores do tipo hidrotalcita oligovanadato pilarizada foram sintetizados pelo método de coprecipitação sob $\mathrm{pH}$ variável, a partir da mistura das soluções de nitrato de magnésio, nitrato de alumínio, hidróxido de sódio e vanadato de sódio. Os ânions

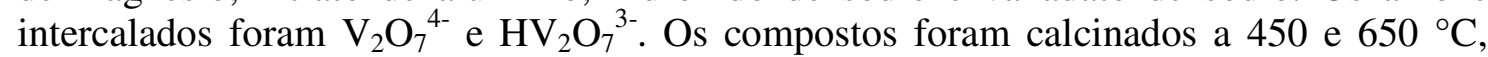
resultando nos óxidos mistos $\mathrm{V}-\mathrm{Mg}$-Al. Estes óxidos são promissores na catálise heterogênea por apresentarem área específica elevada, propriedades básicas e ácidas, estabilidade térmica e mistura homogênea dos óxidos. Os compostos foram caracterizados por TG/DTG, DRX e volumetria de nitrogênio, e as propriedades ácidas e/ou básicas foram avaliadas na reação de decomposição do isopropanol. As análises de DRX dos precursores mostraram a formação de compostos tipo hidrotalcita. Para os catalisadores, houve a formação de $\mathrm{MgO}, \mathrm{V}_{3} \mathrm{O}_{7}, \mathrm{MgAl}_{2} \mathrm{O}_{4}$, entre outros óxidos. Concluiu-se que a introdução de óxidos de vanádio na estrutura dos HDLs aumentou significativamente a atividade dos catalisadores, avaliada pela decomposição do isopropanol.
\end{abstract}

\section{INTRODUÇÃO}

A demanda crescente de propeno torna necessário o uso de processos alternativos que complementem os tradicionais. A reação de desidrogenação oxidativa do propano (DOP) é uma alternativa atrativa para a produção de propeno, pois é exotérmica e inicia-se em temperaturas relativamente baixas (Blanco, Carrazán, Rives, 2008, Cortez, Fierro, Bañares, 2003). Dentre os catalisadores estudados, os baseados em vanádio suportados são os mais ativos nesta reação.

A hidrotalcita é uma argila aniônica natural composta por ânions carbonato intercalado entre lamelas de hidróxido duplo de magnésio e alumínio. Ela também é chamada de "hidróxido duplo lamelar" (HDL) (Crepaldi, Pavan, Valim, 2000). Este composto apresenta a seguinte fórmula geral:

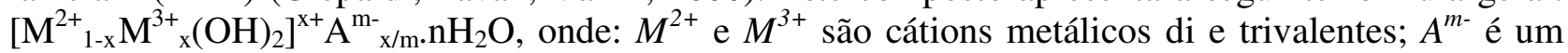
ânion intercalado com carga $m-; x$ é a razão entre os cátions di e trivalentes e $n$ é o número de moléculas de água intercaladas. Algumas das propriedades mais interessantes dos óxidos obtidos pela calcinação dos HDLs são: área superficial elevada; propriedades básicas e formação de misturas homogêneas de óxidos, estáveis em tratamentos térmicos, e a capacidade de troca aniônica, que torna possível a intercalação de outros ânions na estrutura dos HDLs. Estas propriedades tornam este tipo de material favorável para a aplicação em catálise heterogênea (Cavani, Trifirò, Vaccari). 


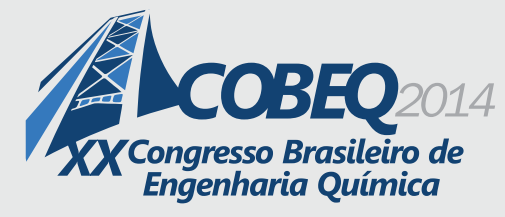

19 a 22 de outubro de 2014

Florianópolis/SC

Bhattacharyya et al. (1995) descreveram a preparação e caracterização de hidrotalcitas oligovanadatos pilarizadas para introduzir uma função de oxidação em argilas. A intercalação de ânions estruturais contendo ânions metálicos em um estado de oxidação elevado (decavanadato) modifica as propriedades catalíticas dos HDLs, devido ao desenvolvimento de sítios ácidos e redox e, em alguns casos, microporosidade interlamelar.

Uma das reações típicas para investigar as propriedades ácidas e/ou básicas dos óxidos mistos é a reação de decomposição do isopropanol (Gervasini, Fenyvesi, Auroux, 1997), a qual conduz às reações de desidratação, formando propeno e éter diisopropílico, e desidrogenação, com formação de acetonas. Os sítios ácidos fortes são responsáveis pela formação de olefinas, enquanto que sítios básicos fortes levam à formação de acetona. Os sítios ácidos e básicos desbalanceados conduzem à formação de ambos os produtos de desidratação. Este trabalho teve como objetivo preparar catalisadores baseados em hidrotalcitas oligovanadatos pilarizadas, para serem utilizados na reação de DOP, e avaliar a influência de dois tipos de ânion interlamelar $\left(\mathrm{V}_{2} \mathrm{O}_{7}{ }^{4-}\right.$ e $\left.\mathrm{HV}_{2} \mathrm{O}_{7}{ }^{3-}\right)$ na reação de decomposição do isopropanol.

\section{EXPERIMENTAL}

\subsection{Síntese dos catalisadores}

Os precursores do tipo hidrotalcita oligovanadato pilarizada foram sintetizados pelo método de coprecipitação a pH variável, segundo o método descrito na literatura (Blasco, Nieto, 1997).

$\mathrm{O}$ primeiro composto, $\mathrm{Mg}_{5} \mathrm{Al}_{2}(\mathrm{OH})_{14}\left(\mathrm{~V}_{2} \mathrm{O}_{7}\right)_{0,5} .5 \mathrm{H}_{2} \mathrm{O}$, foi sintetizado através da adição em gotas de uma solução de $200 \mathrm{~mL}$ contendo $0,2 \mathrm{~mol}$ de $\mathrm{Mg}\left(\mathrm{NO}_{3}\right)_{2} \cdot 6 \mathrm{H}_{2} \mathrm{O}$ e $0,1 \mathrm{~mol}$ de $\mathrm{Al}\left(\mathrm{NO}_{3}\right)_{3} \cdot 9 \mathrm{H}_{2} \mathrm{O}$ a uma segunda solução de $275 \mathrm{~mL}$ contendo 0,1 mol de $\mathrm{NaVO}_{3}$ e $0,7 \mathrm{~mol}$ de $\mathrm{NaOH}$ em pellets, até a obtenção de pH igual a 10, mantendo a agitação constante por uma hora. A mistura gelatinosa foi aquecida a $80^{\circ} \mathrm{C}$ por $15 \mathrm{~h}$, depois de a adição ter sido completada. $\mathrm{O}$ material resfriado a temperatura ambiente foi, então, filtrado, lavado repetidamente com água deionizada e desgaseificada e submetido à secagem em estufa durante $12 \mathrm{~h}$ a uma temperatura de $70^{\circ} \mathrm{C}$. O segundo precursor, $\mathrm{Mg}_{4} \mathrm{Al}_{2}(\mathrm{OH})_{12}\left(\mathrm{HV}_{2} \mathrm{O}_{7}\right)_{0,67} \cdot \mathrm{xH}_{2} \mathrm{O}$, foi preparado de forma semelhante ao descrito anteriormente, porém com a quantidade de hidróxido de sódio igual a $0,6 \mathrm{~mol}$ e com $\mathrm{pH}$ de coprecipitação igual a 8,8. Os compostos obtidos foram calcinados a 450 e $650^{\circ} \mathrm{C}$ por $5 \mathrm{~h}$, obtendo-se assim os catalisadores de óxidos mistos $\mathrm{V}-\mathrm{Mg}$-Al. Os precursores foram designados por $\mathrm{HDL}-\mathrm{MgAl}(\mathrm{X})$, onde $\mathrm{X}$ representa o ânion interlamelar. $\mathrm{E}$ os catalisadores, como $\mathrm{MgAl}(\mathrm{X}) \mathrm{Y}$, onde $\mathrm{Y}$ representa a temperatura de calcinação.

\subsection{Caracterização físico-química dos catalisadores}

As medidas de áreas superficiais, volume de poros e distribuição de volume de poros das amostras dos óxidos mistos foram realizadas em um equipamento da marca BEL, modelo BELSORPmini II, empregando-se a técnica de adsorção-dessorção física de nitrogênio a - $196^{\circ} \mathrm{C}$. Previamente, as amostras foram tratadas in situ sob aquecimento a temperatura de $300^{\circ} \mathrm{C}$ por 3 horas para retirar a água e os gases adsorvidos na superfície e nos poros do sólido. 


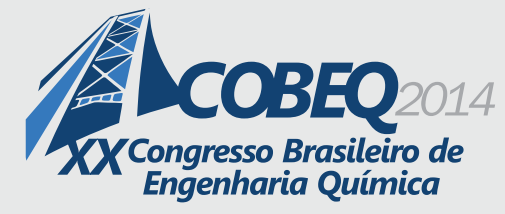

As análises de difração de raios X (DRX) foram realizadas empregando-se o método do pó, utilizando-se um equipamento da marca Seisert, modelo Isodebyefley $1001 \mathrm{com}$ radiação $\mathrm{CuK} \alpha(\lambda=$ $1,54178 \AA$ A) e filtro de $\mathrm{Ni}$. O tubo de raios X foi operado a potência de $40 \mathrm{kV}$ e $30 \mathrm{~mA}$. Os difratogramas de raios $X$ foram obtidos aplicando-se um passo de $2 \theta=0,05^{\circ}$ ( 1 passo por segundo) com $2 \theta$ variando de 5 a $80^{\circ}$. As fases cristalinas foram identificadas com auxílio da biblioteca do JCPDS (Joint Committee of Powder Diffraction Standards).

Os precursores do tipo hidrotalcita foram analisados por termogravimetria (TG/DTG), utilizando-se uma termobalança da marca Shimadzu, modelo TGA-50, com razão de aquecimento de $20{ }^{\circ} \mathrm{C} / \mathrm{min}$, em fluxo de nitrogênio, no intervalo de temperatura de 25 a $900{ }^{\circ} \mathrm{C}$.

As propriedades ácidas e/ou básicas dos catalisadores foram avaliadas através da reação de decomposição do isopropanol, empregando-se um reator de leito fixo (vidro borosilicato) com fluxo contínuo dos reagentes sob pressão atmosférica. Utilizou-se $150 \mathrm{mg}$ do catalisador. A ativação do catalisador foi feita a $450^{\circ} \mathrm{C}$ por $3 \mathrm{~h}$ em fluxo de oxigênio. $\mathrm{O}$ reator foi alimentado com isopropanol $(99,7 \%$, Merck) e injetado na tubulação de alimentação de gases afluentes do reator com auxílio de uma bomba Thermo Separation Products, modelo Spectra P100, à vazão de $0,05 \mathrm{~cm}^{3} / \mathrm{min}$ e diluído em He na vazão de $40 \mathrm{~cm}^{3} / \mathrm{min}$. Os gases efluentes do reator foram analisados por cromatografia gasosa, no qual se utilizou um cromatógrafo do tipo Varian CG-3350, equipado com um detector de condutividade térmica (DCT) e dotado de uma coluna empacotada Carbowax 20M, com rampa de aquecimento entre 30 e $150^{\circ} \mathrm{C}$. Utilizou-se hélio como gás de arraste na coluna. A tubulação do sistema catalítico foi aquecida por uma resistência elétrica para evitar a condensação do reagente e dos produtos da reação. A reação foi avaliada no intervalo de temperatura de 190 a $310^{\circ} \mathrm{C}$ e um fator tempo de residência $\left(\mathrm{W} / \mathrm{F}_{\mathrm{A} 0}\right)$ igual a $2,4 \mathrm{~g} . \mathrm{h} / \mathrm{mol}$. A taxa específica de formação dos produtos (TEP) foi calculada a partir das equações abaixo:

$$
\begin{aligned}
& T E P=\frac{S_{P} \times T R E}{S_{B E T}} \\
& T E R=\frac{X_{A} \times F_{A 0}}{W \times S_{B E T}} \\
& X_{A}(\%)=\left(\frac{n_{R}}{n_{R_{0}}}\right) \times 100 \\
& S_{P}(\%)=\left(\frac{n_{R}}{n_{R_{0}}}\right) \times\left(\frac{N_{C P}}{N_{C R}}\right) \times 100
\end{aligned}
$$

onde TER é a taxa específica de reação; $\mathrm{X}_{\mathrm{A}}$ é a conversão do isopropanol; $\mathrm{S}_{\mathrm{P}}$ a seletividade dos produtos; $n_{R}$ é o número de mols do isopropanol consumido; $n_{R_{0}}$ é o número de mols de isopropanol na alimentação e $N_{C P}$ e $N_{C R}$ são os números de átomos de carbono presentes nos produtos formados e no isopropanol, respectivamente. 


\section{RESULTADOS E DISCUSSÕES}

Análises de TG/DTG foram realizadas com o objetivo de verificar as etapas de degradação térmica dos catalisadores obtidos pela calcinação dos precursores. As curvas TG/DTG para o HDL$\operatorname{MgAl}\left(\mathrm{V}_{2} \mathrm{O}_{7}\right)$ e $\mathrm{HDL}-\mathrm{MgAl}\left(\mathrm{HV}_{2} \mathrm{O}_{7}\right)$ estão representadas na Figura 1 e 2, respectivamente.

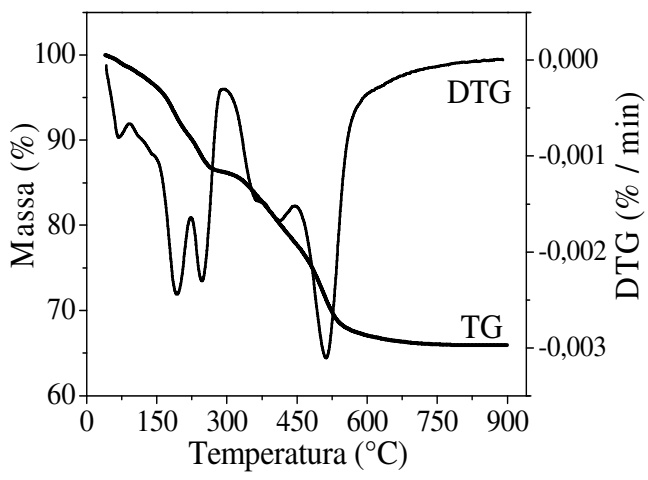

Figura 1 - Curvas TG/DTG para o HDL$\operatorname{MgAl}\left(\mathrm{V}_{2} \mathrm{O}_{7}\right)$.

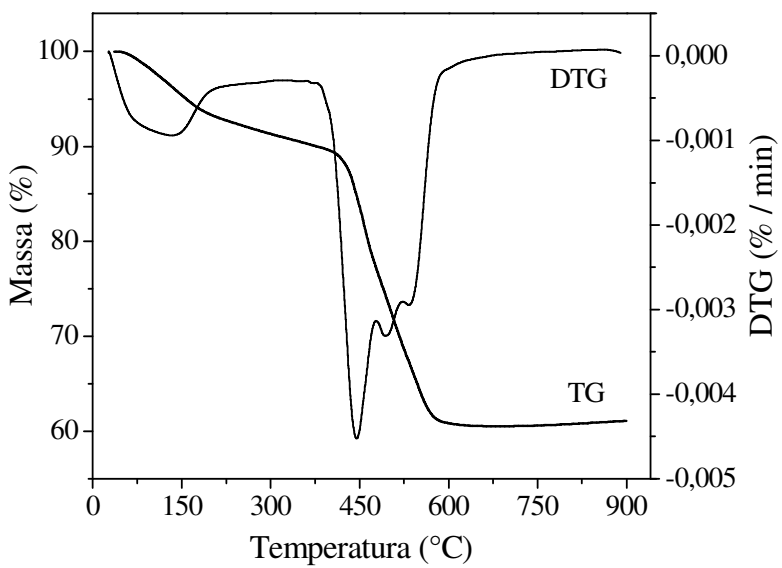

Figura 2 - Curvas TG/DTG para o HDL$\operatorname{MgAl}\left(\mathrm{HV}_{2} \mathrm{O}_{7}\right)$

Pela análise das curvas TG/DTG, foram observadas três etapas de decomposição térmica para o composto HDL- $\mathrm{MgAl}\left(\mathrm{V}_{2} \mathrm{O}_{7}\right)$. Em uma primeira etapa (até $200^{\circ} \mathrm{C}$ ), ocorreu perda de massa de $8,06 \%$, correspondente à perda de água interlamelar e da água adsorvida na superfície. Entre 200 e $522^{\circ} \mathrm{C}$, houve perda de massa de $21,78 \%$, pela qual ocorre a desidroxilação, formando um oxi-hidróxido duplo. Em uma faixa de temperatura de 522 até $800{ }^{\circ} \mathrm{C}$ ocorreu uma perda de massa de $4,24 \%$, devido às transformações dos oxovanadatos interlamelares e decomposição do restante das hidroxilas, formando óxidos mistos $\mathrm{V}-\mathrm{Mg}$-Al, com colapso da estrutura lamelar (Frost et al, 2005). Por outro lado, o $\mathrm{LDH}-\mathrm{MgAl}\left(\mathrm{HV}_{2} \mathrm{O}_{7}\right)$ apresentou em torno de $9 \%$ de perda de massa a mais do que a perda teórica, isto sugere que esta amostra apresentou uma composição química diferente da composição prevista. Esta perda de $9 \%$ pode estar relacionada a uma quantidade maior de moléculas de água presentes na amostra, ou ainda à presença de moléculas de $\mathrm{CO}_{2}$, pois, durante a reação de síntese, os LDHs podem absorver $\mathrm{CO}_{2}$ ao entrar em contato com o ar atmosférico (Crepaldi, Valim, 1998).

As Figuras 3 e 4 apresentam os difratogramas de raios $X$ dos precursores e catalisadores obtidos

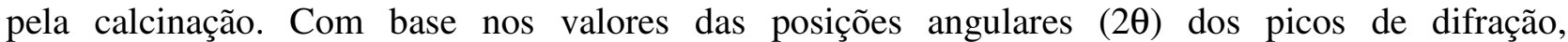
identificados pela Ficha JCPDS (13) $\mathrm{n}^{\circ} 22-700$, pode-se observar a presença de fases cristalinas tipo hidrotalcita (Figura 3). Os três primeiros picos da fase hidrotalcita (ou HDL) correspondem aos planos de difração (003), (006) e (012), respectivamente. Eles são provenientes da difração sobre as camadas de hidróxidos da hidrotalcita (Zheludkevih, Poznyak, Rodrigues, 2010). O primeiro plano é utilizado para calcular o espaçamento basal dos HDLs. 


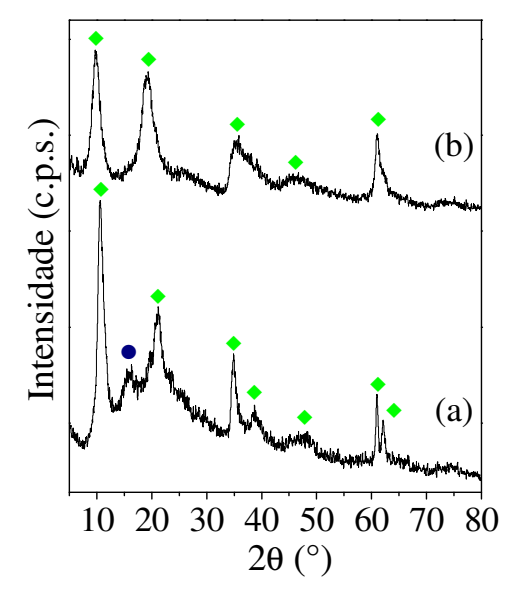

Figura 3 - Difratogramas de raios $\mathrm{X}$ dos precursores: (a) $\mathrm{HDL}-\mathrm{MgAl}\left(\mathrm{V}_{2} \mathrm{O}_{7}\right)$ e (b) $\mathrm{HDL}-\mathrm{MgAl}\left(\mathrm{HV}_{2} \mathrm{O}_{7}\right) .(\diamond)$ hidrotalcita; $(\bullet)$ pico não identificado.
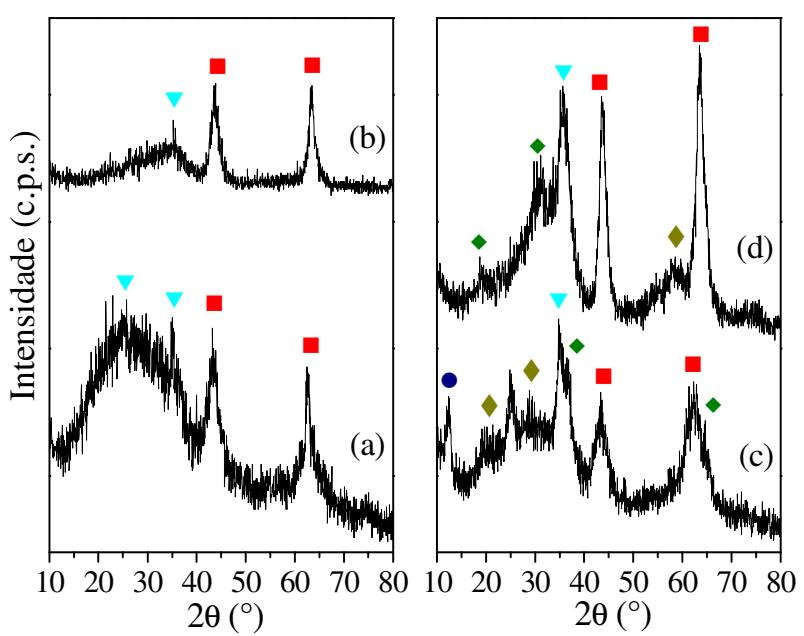

Figura 4 - Difratogramas de raios $\mathrm{X}$ dos catalisadores: (a) $\mathrm{MgAl}\left(\mathrm{V}_{2} \mathrm{O}_{7}\right) 450$, (b)

$\mathrm{MgAl}\left(\mathrm{V}_{2} \mathrm{O}_{7}\right) 650$, (c) $\mathrm{MgAl}\left(\mathrm{HV}_{2} \mathrm{O}_{7}\right) 450$ e (d) $\mathrm{MgAl}\left(\mathrm{HV}_{2} \mathrm{O}_{7}\right) 650$. (口) $\mathrm{MgO} ;(\nabla) \mathrm{V}_{3} \mathrm{O}_{7} ;(\diamond) \mathrm{V}_{2} \mathrm{O}_{5}$;

$(\diamond) \mathrm{MgAl}_{2} \mathrm{O}_{4} ;(\bullet)$ pico não identificado.

Para o HDL-MgAl $\left(\mathrm{V}_{2} \mathrm{O}_{7}\right)$, Figura 3a, o espaçamento basal encontrado foi igual a 8,33 ̊́ e para o HDL-MgAl $\left(\mathrm{HV}_{2} \mathrm{O}_{7}\right)$, Figura 3b, foi 9,07 ̊́. Para a hidrotalcita natural, este valor é 7,69 ̊̊ (Carja, Delahay, 2004). O aumento do espaçamento basal dos precursores em relação à hidrotalcita natural indica que houve a intercalação dos íons vanadatos na estrutura dos compostos sintetizados. Após a calcinação dos precursores, pode observar-se o colapso da estrutura lamelar, com a formação de óxidos mistos com estruturas quase amorfas, identificados pelos difratogramas de raios $\mathrm{X}$ dos catalisadores (Figura 4). Com o aumento da temperatura de calcinação, os picos dos difratogramas se tornaram mais estreitos e intensos, indicando que ocorreu um rearranjo da estrutura dos materiais (Mitik-Dineva, 1998). O composto $\mathrm{MgAl}\left(\mathrm{V}_{2} \mathrm{O}_{7}\right) 450$ (Figura 4a) apresentou dois picos em $2 \theta=43,42$ e $62,65^{\circ}$, que são atribuídos ao periclásio ( $\left.\mathrm{MgO}\right)$. A Ficha JCPDS do $\mathrm{MgO}\left(\mathrm{n}^{\circ}\right.$ 4-829) apresenta valores um pouco distintos dos resultados experimentais $\left(2 \theta=42,95\right.$ e $\left.62,36^{\circ}\right)$, indicando que na presença de alumínio um defeito ocorre na estrutura do periclásio, formando uma solução sólida comumente chamada de magnésia-alumina (Blanco, Carrazán, Rives, 2008). Para este mesmo composto, também foram observados dois picos em $2 \theta=25,05$ e $35,08^{\circ}$, os quais, segundo a Ficha ${ }^{\circ}$ 27-940, pertencem ao óxido de vanádio $\mathrm{V}_{3} \mathrm{O}_{7}$ (composto estequiométrico intermediário entre $\mathrm{VO}_{2} \mathrm{e}$ $\mathrm{V}_{2} \mathrm{O}_{5}$ ). $\mathrm{O} \mathrm{MgAl}\left(\mathrm{V}_{2} \mathrm{O}_{7}\right) 650$ (Figura $4 \mathrm{~b}$ ) também apresentou picos referentes às fases de $\mathrm{MgO}$ e de $\mathrm{V}_{3} \mathrm{O}_{7}$. Assim como o $\mathrm{MgAl}\left(\mathrm{HV}_{2} \mathrm{O}_{7}\right) 450$ (Figura 4c), que além destes, apresentou picos da fase $\mathrm{MgAl}_{2} \mathrm{O}_{4}$ - espinélio (Ficha $\mathrm{n}^{\circ}$ 21-1152) e um pico que não foi identificado. Enquanto que o $\mathrm{MgAl}\left(\mathrm{HV}_{2} \mathrm{O}_{7}\right) 650$ (Figura 4d), obteve picos de $\mathrm{MgO}, \mathrm{MgAl}_{2} \mathrm{O}_{4}, \mathrm{VO}_{2}\left(\right.$ Ficha $\mathrm{n}^{\circ}$ 31-1439) e $\mathrm{V}_{2} \mathrm{O}_{5}$ (Ficha ${ }^{\circ}$ 9-387). 


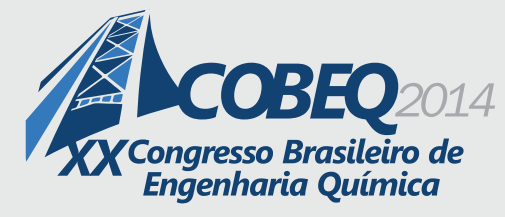

19 a 22 de outubro de 2014

Florianópolis/SC

Pela Tabela 1, podem ser analisados os resultados de área superficial $\left(\mathrm{S}_{\mathrm{BET}}\right)$ e volume de poros $\left(\mathrm{V}_{\mathrm{p}}\right)$ dos catalisadores, obtidos pelos métodos BET (Brunauer, Emmett e Teller) e BJH (Barret, Joyer e Halenda), respectivamente.

Tabela 1 - Valores obtidos de área superficial específica $\left(\mathrm{S}_{\mathrm{BET}}\right)$ e volume específico de poros $\left(\mathrm{V}_{\mathrm{p}}\right)$ dos catalisadores.

\begin{tabular}{ccc}
\hline Catalisadores & $\mathrm{S}_{\mathrm{BET}}\left(\mathrm{m}^{2} / \mathrm{g}\right)$ & $\mathrm{V}_{\mathrm{p}}\left(\mathrm{cm}^{3} / \mathrm{g}\right)$ \\
\hline $\operatorname{MgAl}\left(\mathrm{V}_{2} \mathrm{O}_{7}\right) 450$ & 98 & 0,49 \\
$\operatorname{MgAl}\left(\mathrm{V}_{2} \mathrm{O}_{7}\right) 650$ & 101 & 0,51 \\
$\operatorname{MgAl}\left(\mathrm{HV}_{2} \mathrm{O}_{7}\right) 450$ & 24 & 0,09 \\
$\operatorname{MgAl}\left(\mathrm{HV}_{2} \mathrm{O}_{7}\right) 650$ & 35 & 0,17 \\
\hline
\end{tabular}

Os catalisadores $\operatorname{MgAl}\left(\mathrm{V}_{2} \mathrm{O}_{7}\right) 450$ e $\operatorname{MgAl}\left(\mathrm{V}_{2} \mathrm{O}_{7}\right) 650$ apresentaram valores de $\mathrm{S}_{\mathrm{BET}}$ e $\mathrm{V}_{\mathrm{p}}$ constantes, em média $100 \mathrm{~m}^{2} / \mathrm{g}$ e $0,50 \mathrm{~cm}^{3} / \mathrm{g}$, respectivamente. O composto $\mathrm{MgAl}\left(\mathrm{HV}_{2} \mathrm{O}_{7}\right) 650$ obteve valores de $\mathrm{S}_{\mathrm{BET}}$ e $\mathrm{V}_{\mathrm{p}}$ ligeiramente superiores em relação ao $\mathrm{MgAl}\left(\mathrm{HV}_{2} \mathrm{O}_{7}\right) 450$, conforme mostrados na Tabela 1. Os catalisadores $\mathrm{MgAl}\left(\mathrm{V}_{2} \mathrm{O}_{7}\right) \mathrm{Y}$ obtiveram maiores resultados de $\mathrm{S}_{\mathrm{BET}}$ e $\mathrm{V}_{\mathrm{p}}$ do que os $\operatorname{MgAl}\left(\mathrm{HV}_{2} \mathrm{O}_{7}\right) \mathrm{Y}$.

Com a finalidade de se obter um parâmetro para a avaliação da influência do vanádio na reação de decomposição do isopropanol, foi realizado um teste catalítico para o catalisador sem vanádio, $\operatorname{MgAl}\left(\mathrm{NO}_{3}\right) 450$. Esta amostra obteve uma conversão de isopropanol em torno de $2,35 \%$, para a temperatura de $280^{\circ} \mathrm{C}$. Para os catalisadores com vanádio, a conversão nesta temperatura foi significativamente maior, como pode ser observado na Tabela 2.

Tabela 2 - Conversão do isopropanol $\left(\mathrm{X}_{\mathrm{A}}\right)$, taxa específica de reação (TER) e taxa específica de formação dos produtos (TEP) referentes à temperatura de $280^{\circ} \mathrm{C}$, para cada catalisador.

\begin{tabular}{ccccc}
\hline \multirow{2}{*}{ Catalisadores } & $\mathrm{X}_{\mathrm{A}}$ & $\begin{array}{c}\text { TER } \\
(\%)\end{array}$ & \multicolumn{2}{c}{$\begin{array}{c}\text { TEP } \\
\left(\mu \mathrm{mol} / \mathrm{m}^{2} \mathrm{~min}\right)\end{array}$} \\
\cline { 4 - 5 } & & & Propeno & Éter diisopropílico \\
\hline $\operatorname{MgAl}\left(\mathrm{V}_{2} \mathrm{O}_{7}\right) 450$ & 9,09 & 4,06 & 3,62 & 2,57 \\
$\operatorname{MgAl}\left(\mathrm{V}_{2} \mathrm{O}_{7}\right) 650$ & 7,58 & 3,29 & 2,81 & 0,49 \\
$\operatorname{MgAl}\left(\mathrm{HV}_{2} \mathrm{O}_{7}\right) 450$ & 12,92 & 23,54 & 16,95 & 6,59 \\
$\operatorname{MgAl}\left(\mathrm{HV}_{2} \mathrm{O}_{7}\right) 650$ & 6,24 & 8,20 & 6,75 & 1,34 \\
\hline
\end{tabular}

Os produtos de desidratação do isopropanol são formados a partir de três mecanismos de eliminação $\left(E_{\mathrm{i}}\right): E_{1}, E_{1 B}$ e $E_{2}$. A formação de propeno pode ocorrer pelo mecanismo $E_{1}$, através de sítios ácidos fortes de Lewis ou Brønsted. Este produto pode ser formado também pelo mecanismo $\mathrm{E}_{1 \mathrm{~B}}$, o qual necessita de forças de sítios ácidos e básicos desbalanceadas, ou seja, sítios básicos fortes e sítios ácidos fracos de Lewis. $\mathrm{E}$ principalmente pelo mecanismo $\mathrm{E}_{2}$, através de sítios ácidos e básicos, ambos de força média ou alta, produzindo concomitantemente éter diisopropílico. Pelo mecanismo $\mathrm{E}_{1 \mathrm{~B}}$ também ocorre a reação de desidrogenação do isopropanol, formando acetona, através 


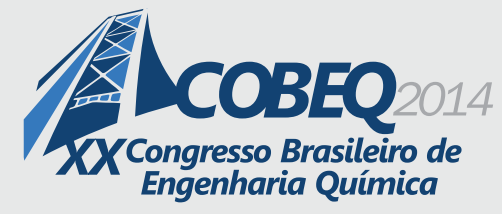

19 a 22 de outubro de 2014

Florianópolis/SC

de sítios básicos fortes e tendo carbânions como intermediários (Gervasini, Fenyvesi, Auroux, 1997). Observou-se a formação de propeno e éter diisopropílico sobre sítios ácidos e básicos fortes e apenas traços de acetona sobre sítios básicos. A maior atividade quanto à formação de produtos de desidratação (propeno e éter) em relação ao produto de desidrogenação (acetona) pode ser explicada não somente pela força, mas também pela densidade dos sítios ácidos e/ou básicos, principalmente pelos sítios ácidos, gerados pelo V e Al (Youssef, Khalil, Girgis, 1992). Embora áreas superficiais elevadas sejam favoráveis à atividade catalítica, os catalisadores com maior $S_{\text {BET }}$ e $V_{p}$ (Tabela 1), intercalados com o ânion $\mathrm{V}_{2} \mathrm{O}_{7}^{4-}$, foram os que apresentaram menor atividade (Tabela 2).

Pelos difratogramas de raios $\mathrm{X}$ (Figura 3), verifica-se a presença das fases de $\mathrm{MgO}$ e $\mathrm{V}_{3} \mathrm{O}_{7}$ nestes compostos. Enquanto que os catalisadores com menor $S_{\text {BET }}$ e $V_{p}$, intercalados com o ânion $\mathrm{HV}_{2} \mathrm{O}_{7}{ }^{3-}$, foram os mais ativos. Estes apresentaram a formação de $\mathrm{MgO}, \mathrm{MgAl}_{2} \mathrm{O}_{4}$ e diferentes espécies de óxidos de vanádio, os quais provavelmente favoreceram a atividade catalítica. Segundo a literatura (Youssef, Khalil, Girgis, 1992), nenhuma relação regular tem sido observada entre a decomposição do isopropanol e a extensão da área específica da superfície, assim como em relação ao volume total de poros dos catalisadores. A acidez é a propriedade mais importante dos óxidos estequiométricos, tais como $\mathrm{MgO}$ e $\mathrm{MgAl}_{2} \mathrm{O}_{4}$, em relação à atividade catalítica, sendo esta proporcional ao aumento da acidez da superfície do catalisador. Deste modo, considerou-se que esta propriedade teve mais influência na decomposição do isopropanol do que a área superficial do catalisador.

\section{CONCLUSÕES}

Os dois precursores sintetizados pelo método de coprecipitação apresentaram a formação de compostos do tipo hidrotalcita. Após a calcinação, formaram-se catalisadores de óxidos mistos V-MgAl, conforme as análises de DRX e TG/DTG.

Os catalisadores contendo o ânion $\mathrm{HV}_{2} \mathrm{O}_{7}{ }^{3-}$ no domínio interlamelar apresentaram menor área específica e volume de poros, porém foram os mais ativos na decomposição do isopropanol, em relação aos compostos contendo o ânion $\mathrm{V}_{2} \mathrm{O}_{7}^{4-}$.

Fazendo uma comparação entre os resultados de conversão dos compostos contendo vanádio e do composto sem vanádio, conclui-se que a introdução de óxidos de vanádio na estrutura dos HDLs teve uma influência significativa na reação de decomposição do isopropanol, quanto à produção de propileno.

\section{AGRADECIMENTOS}

A CAPES, pela bolsa outorgada a Flávia de A. Cipolli e aos professores da EEL-USP, Paulo Suzuki e Maria Lúcia C. P. da Silva, pelas análises de DRX e TG/DTG, respectivamente. 


\section{REFERÊNCIAS}

BHATTACHARYYA, A.; HALL, D. B.; BARNES, T. J. Novel oligovanadate-pillared hydrotalcites. Applied Clay Science, v.10, p.57-67, 1995.

BLANCO, S.; CARRAZÁN, S. R. G.; RIVES, V. Oxidative dehydrogenation of propane on Mg-VAl mixed oxides. Applied Catalysis A: General, v. 342, p. 93-98, 2008.

BLASCO, T.; NIETO, J. M. Oxidative dehydrogenation of short chain alkanes on supported vanadium oxide catalysts , Applied Catalysis A: General, v 157, p. 117-142, 1997.

CARJA, G.; DELAHAY, G. Mesoporous mixed oxides derived from pillared oxovanadates layered double hydroxides as new catalysts for the selective catalytic reduction of $\mathrm{NO}$ by $\mathrm{NH}_{3}$. Applied Catalysis B: Environmental, v. 47, p. 59-66, 2004.

CAVANI, F.; TRIFIRÒ, F.; VACCARI, A. Hydrotalcite-type anionic clays: preparation, properties and applications. Catalysis Today, v. 11, p. 173-301, 1991.

CORTEZ, G.G.; FIERRO, J.L.G.; BAÑARES, M.A., Role of potassium on the structure and activity of alumina-supported vanadium oxide catalysts for propane oxidative dehydrogenation. Catalysis Today, v. 78, p. 219-228, 2003.

CREPALDI, E. L.; PAVAN, P. C.; VALIM, J. B. Comparative study of the coprecipitation methods for the preparation of layered double hydroxides. Journal Brazilian Chemical Society, v. 11, n. 1, p. 64-70, 2000.

FROST, R. L; MUSUMECI, A. W.; BOSTROM, T.; ADEBAJO, M. O.; WEIER, M. L.; MARTENS, W. Thermal decomposition of hydrotalcite with chromate, molybdate or sulphate in the interlayer. Thermochimica Acta, v. 429, p. 179-187, 2005.

GERVASINI, A.; FENYVESI, J.; AUROUX, A. Study of the acidic character of modified metal oxide surfaces using the test of isopropanol decomposition. Catalysis Letters, v. 43, p. 219-228, 1997.

MITIK-DINEVA, N.; WANG, J.; TRUONG, V. K.; STODDART, P.; MALHERBE, F.; CRAWFORD, R. J. Hydrotalcite-like compounds as precursors for mixed oxides catalysts in the oxydehydrogenation of ethylbenzene. Applied Clay Science, v. 13, p. 381-399, 1998.

YOUSSEF, A. M.; KHALIL, L. B.; GIRGIS, B. S. Decomposition of isopropanol on magnesium oxide/silica in relation to texture, acidity and chemical composition. Applied Catalysis A: General, v. 81, p. 1-13, 1992.

ZHELUDKEVICH, M. L.; POZNYAK, L. M.; RODRIGUES, D. Active protection coatings with layered double hydroxide nanocontainers of corrosion inhibitor. Corrosion Science, v. 52, p. 602-611, 2010. 